Published in Medical Hypotheses 57(5): 619-627 (2001)

\title{
Mechanism of Anesthetic Action: Oxygen Pathway Perturbation Hypothesis
}

\author{
Huping Hu \\ Biophysics Consulting Group \\ 32 Michael Drive \\ Old Bethpage, NewYork 11804 \\ E-mail: drhu@worldnet.att.net
}

\author{
Maoxin Wu \\ Department of Pathology \\ The Mount Sinai Medical Center \\ One Gustave L. Levy Place \\ New York, New York 10029-6574
}

(Dated: January 23, 2001)

\begin{abstract}
Although more than 150 years have past since the discovery of general anesthetics, how they precisely work remains a mystery. We propose a novel unitary mechanism of general anesthesia verifiable by experiments. In the proposed mechanism, general anesthetics perturb oxygen pathways in both membranes and oxygen-utilizing proteins such that the availabilities of oxygen to its sites of utilization are reduced which in turn triggers cascading cellular responses through oxygen-sensing mechanisms resulting in general anesthesia. Despite the general assumption that cell membranes are readily permeable to oxygen, exiting publications indicate that these membranes are plausible oxygen transport barriers. The present hypothesis provides a unified framework for explaining phenomena associated with general anesthesia and experimental results on the actions of general anesthetics. If verified by experiments, the proposed mechanism also has other significant medical and biological implications.
\end{abstract}




\section{INTRODUCTION}

General anesthetics affect a variety of ion channels and neurotransmitter receptor subtypes (1-7). They even affect actin-based motility in dendritic spines (8). However, how they precisely work remains a mystery. There are mainly two schools of thoughts on how they work but neither is universally accepted.

The first and the oldest is the "lipid theory" which proposes that anesthetics dissolve into cell membranes and produce common structural perturbation resulting in depressed function of ion channels and receptors that are involved in brain functions (9-14). However, there has been no direct experimental evidence to support the notion that anesthetic perturbation of membrane depresses functions of membrane proteins.

The second, more popular and recent theory is the "protein theory" which suggests that anesthetics directly interact with membrane proteins such as ion channels and receptors that are involved in brain functions (15-17). But again there is no direct experimental evidence, e.g., that obtained by applying any of the established essays, such as radioligand-binding essay, to support the notion that general anesthetics specifically bind to these ion channels and receptors. In addition, this theory doesn't seem to square well with the low affinity and diversity of the general anesthetics.

Overall, it seems that each theory has its merits and may be partially correct. The lipid theory may be correct on the notion that membrane perturbation is related to anesthesia but incorrect on the notion that membrane perturbation depresses the functions of ion channels and receptors involved in brain functions. On the other hand, the protein theory may be correct on the notion that specific bindings of certain substances on ion channels and receptors involved in brain functions are related to anesthesia but incorrect on the notion that general anesthetics specifically bind to these proteins. Therefore, finding the missing link between these two types of theories may be the key to solve this 150-year old medical mystery.

We postulate that the diverse effects of general anesthetics observed at the cellular level (see 1-7) are all cascading cellular responses triggered by the change in the availability, at molecular level, of a biochemical component essential to consciousness and other brain functions which in turn is caused by the anesthetic perturbations of the pathways of that component in both membranes and proteins.

We further speculate that oxygen is such a biochemical component because it provides one of the essential components necessary for energy production in the brain cells. The most immediate purpose of our respiratory and circulatory systems is to deliver oxygen to the tissue for use by mitochondria, and to eliminate carbon dioxide. Further, although the brain represents only $2 \%$ of the body weight, it receives $15 \%$ of the cardiac output, $20 \%$ of total oxygen consumption, and $25 \%$ of total body glucose utilization (18).

The transport of oxygen from the brain tissue to the mitochondria in the brain cells, where it is consumed and energy is produced, is presumably governed by the physical law of diffusion (19). As discussed later, contrary to the general assumption that cell membranes are readily permeable to oxygen, existing publications support the notion that these membranes are plausible oxygen transport barriers. The lateral movement of oxygen within the membrane to oxygen-utilizing protein may also encounter barriers. Further, the movement of oxygen in the protein to its site of utilization such as movement in a hydrophobic 
pocket may also encounter barriers. Since both molecular oxygen and general anesthetics are hydrophobic, we speculate that the latter perturb the pathways of the former in both membranes and proteins.

In essence, we postulate that general anesthetics perturb oxygen pathways in both membranes and proteins such that its availabilities to the sites of utilization are reduced which in turn triggers cascading cellular responses through oxygen-sensing mechanisms resulting in general anesthesia.

The present hypothesis provides a unified framework for explaining phenomena associated with general anesthesia and experimental results on the actions of general anesthetics. If valid, the proposed mechanism also has other significant medical and biological implications.

\section{PHYSICS OF OXYGEN TRANSPORT}

Movement of small neutral molecules such as $\mathrm{O}_{2}$ across membranes typically occurs by diffusion, a form of random motion powered by thermal vibrations (19). $\mathrm{O}_{2}$ has to diffuse through the plasma membrane and the outer and inner membranes of the mitochondria before it can reach the mitochondrial matrix. (Fig.1a).

At each membrane, $\mathrm{O}_{2}$ has to diffuse through a first polar surface region, a hydrophobic region and a second polar surface region (Fig.1b). The free energy barrier for $\mathrm{O}_{2}$ uptake is also schematically shown in Figure 1b. Thus, although $\mathrm{O}_{2}$ may diffuse through the hydrophobic region without too much difficulty, it needs much more thermal energy to diffuse through the polar surface region at each side of the membrane (19).

For a membrane separating two solution of concentration $\left[\mathrm{O}_{2}\right]_{1}{ }^{\mathrm{aq}}$ and $\left[\mathrm{O}_{2}\right]_{2}{ }^{\mathrm{aq}}$, where $\left[\mathrm{O}_{2}\right]_{1}{ }^{\mathrm{aq}}>\left[\mathrm{O}_{2}\right]_{2}{ }^{\mathrm{aq}}$ (Figure $1 \mathrm{~b}$ ), the $\mathrm{O}_{2}$ diffusion rate across the membrane is given by the modified Fick's law:

$$
\mathrm{J}=\mathrm{P}\left(\left[\mathrm{O}_{2}\right]_{1}{ }^{\mathrm{aq}}-\left[\mathrm{O}_{2}\right]_{2}{ }^{\mathrm{aq}}\right)
$$

where $\mathrm{P}$ is the $\mathrm{O}_{2}$ permeability coefficient of the membrane. It is proportional to $\mathrm{O}_{2}$ partition coefficient $\mathrm{K}$ and diffusion coefficient $\mathrm{D}$ of the membrane and is inversely proportional to membrane thickness $\mathrm{x}(20)$, or

$$
\mathrm{P}=\mathrm{K}(\mathrm{D} / \mathrm{x})
$$

Thus, the diffusion rate of $\mathrm{O}_{2}$ across the membrane is proportional to its diffusion coefficient $\mathrm{D}$ and partition coefficient $\mathrm{K}$. It follows that if a substance significantly reduces $\mathrm{D}$ and/or $\mathrm{K}$ it may cause hypoxia or oxygen-limiting situations to develop at cellular or sub-cellular level.

In a simplified model, one can directly relate $\mathrm{O}_{2}$ permeability coefficient P of a membrane to the percentage of "holes" on the membrane (21). To do this one treats the membrane as an infinitely thin reflecting wall with homogeneously distributed holes. Further, let $\alpha$ be the ratio of the total surface of all holes to the membrane surface (21). It can be shown then: 


$$
\mathrm{J}=\alpha(1-\alpha)^{-1}\left[\left(\mathrm{k}_{\mathrm{b}} \mathrm{T}\right)(2 \pi \mathrm{m})^{-1}\right]^{1 / 2}\left(\left[\mathrm{O}_{2}\right]_{1}{ }^{\mathrm{aq}}-\left[\mathrm{O}_{2}\right]_{2}{ }^{\mathrm{aq}}\right)
$$

Compare equation [3] to equation [1], one gets:

$$
\mathrm{P}=\alpha(1-\alpha)^{-1}\left[\left(\mathrm{k}_{\mathrm{b}} \mathrm{T}\right)(2 \pi \mathrm{m})^{-1}\right]^{1 / 2}
$$

where $\mathrm{T}, \mathrm{k}_{\mathrm{b}}$ and $\mathrm{m}$ denote temperature, Boltzmann constant and mass of $\mathrm{O}_{2}$ respectively (21).

Thus, in the simplified model, the diffusion rate of $\mathrm{O}_{2}$ across the membrane is directly related to the percentage of holes on the membrane. It follows that, if a substance significantly reduces $\alpha$, it may cause hypoxia or oxygen-limiting situations to develop at cellular or sub-cellular level.

The lateral movement of $\mathrm{O}_{2}$ within the membrane, such as the inner membrane of the mitochondria, may also be involved before $\mathrm{O}_{2}$ can reach the $\mathrm{O}_{2}$ utilizing protein (see Fig.2), such as the cytochrome oxidase complex of the Creb's cycle. The lateral diffusion rate $\mathrm{J}_{\mathrm{m}}$ of $\mathrm{O}_{2}$ is presumably subject to the Fick's law:

$$
\mathrm{J}_{\mathrm{m}}=\left(\mathrm{D}_{\mathrm{m}} / \mathrm{d}\right)\left(\left[\mathrm{O}_{2}\right]_{1}{ }^{\mathrm{m}}-\left[\mathrm{O}_{2}\right]_{2}{ }^{\mathrm{m}}\right)
$$

where $\left[\mathrm{O}_{2}\right]_{1}{ }^{\mathrm{m}}$ and $\left[\mathrm{O}_{2}\right]_{2}{ }^{\mathrm{m}}$ are respectively the $\mathrm{O}_{2}$ concentrations in two regions of the membrane $\left(\left[\mathrm{O}_{2}\right]_{1}{ }^{\mathrm{m}}>\left[\mathrm{O}_{2}\right]_{2}{ }^{\mathrm{m}}\right), \mathrm{D}_{\mathrm{m}}$ is the lateral diffusion coefficient of $\mathrm{O}_{2}$ in the membrane, and $\mathrm{d}$ is the distance separating the two regions of the membrane.

Thus, the lateral diffusion rate of oxygen within membrane is proportional to its diffusion coefficient $\mathrm{D}_{\mathrm{m}}$. It follows that if a substance significantly reduces $\mathrm{D}_{\mathrm{m}}$ oxygen-limiting situations may develop near the site of $\mathrm{O}_{2}$ utilization.

Finally, the movement of $\mathrm{O} 2$ within the protein such as movement in a hydrophobic pocket may also be involved before $\mathrm{O}_{2}$ can reach its site of utilization (see Fig.2). Conceivably, any substance which blocks, narrows, distorts or otherwise interferes with the $\mathrm{O}_{2}$ pathway within the protein may reduce oxygen availability to its site of utilization at the molecular level resulting in hypoxia-mimic situations.

\section{MEMBRANE AS OXYGEN DIFFUSION BARRIER}

In view of the above discussions, it is possible that certain biological membranes are oxygen diffusion barriers because of their particular structurecomposition. Neurons are highly irregular cells with a particular membrane structure-composition. It is also possible that certain substances significantly reduce membrane permeability to $\mathrm{O}_{2}$ by reducing $\mathrm{O}_{2}$ diffusion coefficient and/or partition coefficient of the membrane, or, from a different perspective, by reducing the percentage of "holes" on the membrane. Further, it is possible that 
certain substances significantly reduce $\mathrm{O}_{2}$ lateral diffusion coefficient of the membrane or otherwise perturb its availability to $\mathrm{O}_{2}$-utilizing proteins. Indeed, there are publications supporting these speculations as discussed below.

Electron paramagnetic resonance (EPR) studies of $\mathrm{O}_{2}$ transport parameters in phosphatidylcholine-cholesterol membranes showed that the addition of $50 \mathrm{~mol} \%$ cholesterol decreases oxygen permeability of the membrane by a factor of 2.5 to 5 (22). The same study also reported that the main resistance to oxygen transport is located in and near the polar surface regions in the membrane (22). In an earlier study, it was reported that the addition of an equimolar amount of cholesterol into liposomes of egg lecithin decreased the partition coefficient of $\mathrm{CO}_{2}$, a small, neutral molecule like $\mathrm{O}_{2}$, by about $25 \%$ (20).

In another report, EPR studies of $\mathrm{O}_{2}$ transport in the $\mathrm{CHO}$ cell plasma membrane showed that at $37{ }^{\circ} \mathrm{C}$ the oxygen permeability coefficient for the plasma membrane was about two times lower than for a water layer of the same thickness as the membrane (23). Although it was concluded that oxygen permeation across the cell plasma membrane could not be a rate-limiting step for cellular respiration, $\left[\mathrm{O}_{2}\right]$ difference in the nanomolar range across the $\mathrm{CHO}$ cell plasma membrane at physiological conditions does exit (23).

Classically, there is a direct correlation between the hydrophobic nature of a molecule and its rate of permeation across a biological membrane. So cell membranes should be more permeable to small, neutral molecules than they are to charged molecules of similar sizes. However, it was reported that apical membranes of renal tube cells from the medullary thick ascending limb of Henle are virtually impermeable to $\mathrm{NH}_{3}$, a small neutral molecule (24). It was suggested that such low permeability is due to the specific lipid structure-composition of the apical bilayer (24). Thus, since $\mathrm{O}_{2}$ is a small neutral molecule like $\mathrm{NH}_{3}$, it is possible that certain biological membranes such as those of the neurons may have reduced permeability to $\mathrm{O}_{2}$ due to their structure-composition or changes thereof.

Furthermore, it has been repeatedly demonstrated through EPR oxymetry that the measurement of extracellular $\left[\mathrm{O}_{2}\right]$ may not accurately reflect intracellular $\left[\mathrm{O}_{2}\right]$ since the average intracellular $\left[\mathrm{O}_{2}\right]$ are consistently lower than the average extracellular $\left[\mathrm{O}_{2}\right]$ in quite a few cell suspensions under normal or stimulated conditions (25-28). Thus, contrary to the popular notion that $\mathrm{O}_{2}$ freely diffuses through membranes, these publications suggest that biological membranes are plausible oxygen diffusion barriers.

In addition, it was reported that treatment of sickle red cells with certain voltage pulse causes a change in the cell membrane so as to facilitate the permeation of $\mathrm{O}_{2}$ (29). This result also suggests that some membranes may have low $\mathrm{O}_{2}$ permeability and a modification is possible through external influence.

Finally, the study of intracellular supply of $\mathrm{O}_{2}$ to mitochondria by measuring the $\mathrm{O}_{2}$ dependence of oxidation of cytochromes has shown that the $\mathrm{O}_{2}$ diffusion coefficient in the region of mitochondria in situ is considerably small and suggests that a significant $\mathrm{O}_{2}$ gradient in the vicinity of mitochondria occurs under hypoxic conditions (30). These results suggest that the $\mathrm{O}_{2}$ diffusion rate 
may be a critical factor for intracellular $\mathrm{O}_{2}$ supply to mitochondria during hypoxia (30).

\section{ANESTHETIC PERTURBATION OF MEMBRANE}

Both theoretical and experimental studies have shown that many general anesthetics cause changes in membrane structures and properties at or just above the clinical concentrations required for anesthesia (10-14,31).

Fore example, it was reported that, with dipalmitoyl-L- $\alpha$ phosphatidylcholine (DPPC) vesicle membranes, the main transition temperature between the lipid-crystal and solid gel phases decreases by the addition of short chain 1-alkanols, but increases by long-chain l-alkanols (32). The switchover from depression to elevation of the transition temperature occurs at the same carbon-chain length as the cutoff point of anesthetic effects of these 1-alkanols (32). This suggests that the disordering effects of anesthetics on the hydrophobic region of the membrane are closely related to anesthesia and its cutoff point.

It was also reported that while the lipid order parameter reported by nitroxide spin label, 12-doxyl stearate, was decreased by anesthetic alcohols (octanol, decanol, and dodecanol), the non-anesthetic alcohols either did not change it significantly (tetradecanol) or actually increased it (hexadecanol and octadecanol) (12). These results again suggest that the disordering effects of these alcohols on the hydrophobic region of the membrane are closely related to anesthesia and its cutoff point.

Not only general anesthetics perturb hydrophobic regions of membranes, they also perturb the membrane surface regions by disrupting interfacial hydrogen bonding (33). It was reported that the alkanols form hydrogen bonds with the phosphate moiety of DPPC and release the DPPC-bound water (33). These effects increase with the elongation of the carbon chain of 1-alkanols from ethonal $\left(\mathrm{C}_{2}\right)$ to l-decanol $\left(\mathrm{C}_{10}\right)$ and are linearly related to the anesthetic potencies of these alkonols, but suddenly almost disappear at l-tetradecanol $\left(\mathrm{C}_{14}\right)(33)$.

Because of the non-specific nature of anesthetic interactions with membranes and their surface regions, many physical and biochemical parameters may be affected at, or just above, the clinical concentrations (31). The key question is which physical or biochemical parameters play an important role in the production of anesthesia.

\section{ANESTHETIC PERTURBATION OF PROTEIN}

Because of the low affinity and diversity of the general anesthetics, we speculate that their interactions with proteins are mostly in the forms of ligand pathway perturbation, not specific binding. More specifically, we propose that general anesthetics dissolve into hydrophobic regions of protein complex such as 
hydrophobic pockets containing ligand-binding sites thus acting as barriers to the movement of ligand to said sites.

Indeed, most proteins are insensitive to the presence of clinically relevant concentrations of general anesthetics, while some are affected by certain anesthetic agents (34). For example, it was shown that the activity of firefly luciferase could be inhibited by $50 \%$ at surgical concentrations of various anesthetics (34). It was concluded that the inhibition was competitive in nature, with anesthetic molecules competing with substrate luciferin molecules for binding to the enzyme (34).

We speculate that these anesthetics dissolve into the hydrophobic pocket of the enzyme and perturb the oxygen pathway to its site of utilization. Besides luciferin molecules, $\mathrm{O}_{2}$, ATP and $\mathrm{Mg}^{+2}$ are other substrates needed by the enzyme to produce light. It should be pointed out that firefly luciferase is a pure water-soluble protein and has nothing to do with anesthesia.

In another report, it was shown that the n-alcohols $\left(\mathrm{C}_{1}\right.$ to $\left.\mathrm{C}_{14}\right)$ inhibit the membrane-bound enzyme cytochrome c (35). The inhibition constants were shown to correlate well with the number of carbon atoms in the n-alcohols and also their n-octanol/water partition coefficients (35). Thus, it was suggested that these $n$-alcohols inhibit by binding to an octanol-like environment on the enzyme or the protein-phospholipid interface (35).

Again, we speculate that these n-alcohols dissolve into the hydrophobic pocket of cytochrome c oxidase complex containing the site of oxygen utilization thus perturb the oxygen pathway to said site. This alternative explanation is further supported by the finding that neither negatively charged carboxylates nor positively charged amine analogues were observed to cause any inhibition (35), indicating that the region where inhibition took place is probably hydrophobic.

Overall, existing publications suggest that general anesthetics seem to interact with protein (36) in various weak and probably non-specific forms at reasonable pharmacological concentrations. The key question is which interactions are relevant to general anesthesia.

We postulate that general anesthetics perturb oxygen pathways in both membranes and proteins resulting in reduced oxygen availability to its sites of utilization.

\section{OXYGEN PATHWAY PERTURBATION HYPOTHESIS}

The mechanism of general anesthesia according to the present hypothesis is schematically shown in Fig. 3. As soon as general anesthetics are absorbed into brain cells, they immediately act as $\mathrm{O}_{2}$ transport barriers by narrowing, blocking, dislocating, distorting or otherwise interfering with the $\mathrm{O}_{2}$ pathways in both membranes and proteins (Fig. 2) such that its availabilities to the sites of utilization are reduced. The reduced availabilities of $\mathrm{O}_{2}$ may be reflected by hypoxia or hypoxia-mimic situations at cellular or sub-cellular levels. 
In turn, the reductions of $\mathrm{O}_{2}$ availabilities to the sites of utilization activate oxygen-sensing mechanisms of the brain cells through which cascading cellular responses are generated in order to reduce brain energy consumption and preserve brain functionality resulting in general anesthesia.

Therefore, according to the present hypothesis, general anesthesia is the product of brain self-preservation in response to reductions of $\mathrm{O}_{2}$ availabilities to the sites of utilization in brain cells caused by general anesthetics.

If the present hypothesis is correct, the remaining issues need to be resolved are the details of oxygen-sensing mechanisms through which cascading cellular responses are generated. They will probably involve the releases of various modulating messengers and other signaling processes in order to depress/enhance certain excitatory/inhibitory ion channels and receptors involved in brain functions.

\section{OXYGEN-SENSING MECHANISMS}

The mechanisms of $\mathrm{O}_{2}$-sensing have been widely studies (37-41). Hypoxia elicits a variety of physiological and adaptive responses at cellular and sub-cellular levels that requires the existence of an $\mathrm{O}_{2}$ sensor coupled to a signaling system, which in turn activates the functional response (37). Much has been learned about the signaling systems activated by hypoxia, but the nature of the underlying $\mathrm{O}_{2}$-sensor is still unclear (37).

The consensus is that the mitochondrial respiratory chain is in part the site of oxygen sensing (38). They initiate signaling cascades involved in physiological and adaptive responses to hypoxia (38). Further, membrane-bound $\mathrm{NAD}(\mathrm{P}) \mathrm{H}$ oxidase possibly takes part in oxygen sensing (38). It has also been suggested that ion channels may change conductance as a function of [O2], allowing them to signal the onset of hypoxia (41).

The oxygen-sensing mechanisms activated by general anesthetics may or may not be similar to those studied so far.

\section{VERIFICATION OF THE HYPOTHESIS}

The present hypothesis can be verified through several types of experiments described below. Additional supporting evidence will also come from the application of the hypothesis to phenomena associated with anesthesia and experimental results on the actions of general anesthetics as discussed later.

$\underline{\text { Measurement of Intracellular }\left[\mathrm{O}_{2}\right] \text { in vitro }}$ 
According to the present hypothesis, general anesthetics may significantly reduce membrane $\mathrm{O}_{2}$ permeability thus causing cellular or subcellular hypoxia. Therefore, by measuring the effect of anesthetics on intracellular $\left[\mathrm{O}_{2}\right]$ of functional neurons in vitro, one can test the validity of the hypothesis at the cellular level.

There are several methods available for measuring intracellular $\left[\mathrm{O}_{2}\right]$. One of them is the direct insertion of an intracellular $\mathrm{O}_{2}$ electrode into a single cell $(42,43)$. The second method utilizes fluorescence quenching by $\mathrm{O}_{2}$ after the fluorescent probe, pyrenebutyric acid, is taken up by the cells $(44,45)$. The third, more extensively studied method is the EPR oximetry in which the broadening effect of $\mathrm{O}_{2}$ on the intracellular EPR signals of nitroxides is utilized (25-28).

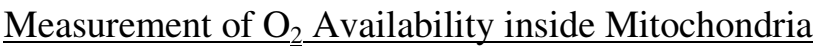

Even if general anesthetics do not cause hypoxia at the cellular level, they may still cause hypoxia or hypoxia-mimic situations inside the mitochondria where oxygen is consumed and energy is produced.

There are several methods available for providing information on the availability of $\mathrm{O}_{2}$ to its sites of utilization inside the mitochondria. One method utilizes the measurement of optical absorption by mitochondrial redox couples $(30,46)$. Another method utilizes the measurement of fluorescence from mitochondrial redox couples $(47,48)$. The authors here suggest yet a third method discussed below.

It has been shown that the cellular reductions of lipid-soluble nitroxides involve the Creb's cycle and are sensitive to the availability of $\mathrm{O}_{2}(49,50)$. Thus, the lower the availability of $\mathrm{O}_{2}$ to its sites of utilization inside the mitochondria, the faster the reduction of a lipid-soluble nitroxide as measured by EPR. It is predicted that if general anesthetics reduce the availability of $\mathrm{O}_{2}$ to its sites of utilization inside the mitochondria, the rate of reduction of a lipid-soluble nitroxide will increase in the presence of general anesthetics at clinically relevant concentrations.

\section{Measurement of $\mathrm{O}_{2}$ transport parameters within the membrane}

The effects of anesthetics on $\mathrm{O}_{2}$ transport in the membranes can be studied by observing, in the presence and absence of general anesthetics, the collision of $\mathrm{O}_{2}$ with nitroxide spin labels placed at various distances from the membrane surface using long-pulse saturation recovery EPR techniques $(22,23)$. The $\mathrm{O}_{2}$ permeability coefficients of the membrane can be estimated from the oxygen transport parameter profiles so obtained $(22,23)$. Further, such profile will also provide information on the effect of general anesthetics on the lateral diffusion of $\mathrm{O}_{2}$ in the membrane. If the present hypothesis is correct, the estimated $\mathrm{O}_{2}$ permeability across the membrane and its lateral diffusion coefficient should decrease in the presence of general anesthetics.

Measurement of Cellular and Sub-cellular $\left[\mathrm{O}_{2}\right]$ in vivo 
There are apparently no suitable methods for directly measuring intracellular $\left[\mathrm{O}_{2}\right]$ in vivo. However, there are various techniques available for measuring brain metabolic rate and energy consumption $(51,52)$ that will provide indirect information on the availability of $\mathrm{O}_{2}$ to its sites of utilization inside the brain cells. The present hypothesis predicts reduced energy consumption and depressed overall brain metabolism. Indeed, supporting publications already exist as discussed below.

\section{APPLICATION OF THE PRESENT HYPOTHESIS}

The present hypothesis provides a unified framework for explaining phenomena associated with general anesthesia and experimental results on the actions of general anesthetics. However, due to limited spaces, only several examples are given below.

One of the important phenomena of general anesthesia is the depression of cerebral metabolic rate (51). According to the present hypothesis, this phenomenon is due to the cascading cellular responses generated through the oxygen-sensing mechanisms activated by anesthetic-induced hypoxia or hypoxiamimic situations at cellular or sub-cellular level. It is well known that hypoxia induces hypometabolism $(34-38,53,54)$.

Secondly, the classical Meyer-Overton correlation that the effectiveness of an anesthetic is closely related to its solubility in lipids (9) can also be explained according to the present hypothesis. The effectiveness of an anesthetic in perturbing the oxygen pathways in membranes and proteins is closely related to its solubility in a hydrophobic environment such as the membrane and the hydrophobic pocket of a protein complex. As the carbon chain of an anesthetic grows, its solubility in the hydrophobic environment increases and so is its effectiveness in perturbing the oxygen pathways in membranes and proteins.

However, when the carbon chain of the anesthetic grow to certain length, it cannot dissolve into an hydrophobic pocket of a protein complex due to its size, nor can it produce disordering effect in the membrane as shown by experiments $(12,32,33)$, thus greatly diminishing its ability to perturb oxygen pathways in membranes and proteins. The end result is the disappearance of the anesthetic effect as its carbon chain grows to certain length. This is known as the anesthesia cut-off phenomenon (see, e.g., 33).

As to the "pressure antagonism" that high pressure reverses anesthesia (55), the present hypothesis can also provide a satisfactory explanation. High pressure minimizes anesthetic perturbation of oxygen pathways in membranes and proteins by respectively squeezing the anesthetics out of the oxygen pathways in the membranes and slowing their movement into the oxygen pathways in the proteins.

The observed effects of general anesthetics on various ion channels and receptors (1-7) at cellular level can all be explained according to the present 
hypothesis as the cascading cellular responses generated through the oxygensensing mechanisms, such as the releases of various modulating messengers, in response to reduced $\mathrm{O}_{2}$ availability to the sites of utilization caused by general anesthetics. Thus, in the proposed mechanism, ion channels and receptors are the direct targets of action by endogenous modulating messengers generated through the oxygen-sensing mechanisms but not general anesthetics themselves.

For example, the widely reported result that anesthetics and $n$-alcohols potentiate the action of the strychinine-sensitive glycine receptor (GlyR) and the so-aminobutyric acid type A $\left(\mathrm{GABA}_{\mathrm{A}}\right)$ (see, e.g., 5, 56) can be satisfactorily explained as the potentiation of the actions of these receptors by the binding of endogenous messengers generated through the oxygen-sensing mechanisms. All reported results on the effects of general anesthetics on other ion channels and receptors involved in brain functions $(1-4,6,7)$ can also be similarly explained.

Further, it was reported recently that inhalational anesthetics block actinbased motility in dendritic spines of the neurons and fibroblasts at clinically effective concentrations but do not influence actin polymerization in vitro (8). Thus, it seems that these anesthetics affect actin dynamics indirectly (8). According to the present hypothesis, the actin-based motility is probably affected through the oxygen-sensing mechanisms such as the release of modulating messengers or the depression of cellular metabolic activities.

There is no direct experimental evidence, e.g., that obtained by applying any of the established essay methods such as radioligand-binding essay, supporting the proposition that anesthesia is the result of direct interactions between anesthetics and the ion channels and receptors involved in brain functions. Even those results obtained by site-directed mutagenesis techniques do not necessarily provide evidence to support such a proposition. Indeed, those results can also be satisfactorily explained according to the present hypothesis.

For example, it has been reported that specific mutations of certain residues of GlyR and $\mathrm{GABA}_{\mathrm{A}}$ receptors can abolish or markedly reduce the effect of anesthetic or ethanol on these receptors without necessarily affecting receptor function seemingly suggesting that anesthetics and alcohols may bind in a cavity located on the receptor complex (56). However, according to the present hypothesis the binding to the above-said cavity by a modulating messenger released through the oxygen-sensing mechanisms in response to anestheticinduced hypoxia or hypoxia-mimic situations can also explained the observed effects. Alternatively, the observed effect can be explained by the binding of the above-said messenger to somewhere else in the receptor complex but the abovesaid cavity provides a transduction or gating site required for the messenger's action.

The only specific binding reported so far is the laboratory-created covalent binding between an anesthetic analog, propanethiol (a sulfhydrylspecific reagent), and cysteine residues introduced into GlyR and $\mathrm{GABA}_{\mathrm{A}}$ receptors through site-directed mutagenesis techniques (57). It was reported that such covalent binding irreversibly enhances receptor functions (57). However, since such binding is artificially created, it does not prove direct binding of 
anesthetics to these receptors. According to the present hypothesis, the observed effect can be explained as the irreversible occupation of the messenger binding site or the messenger-mediated transduction or gating site.

\section{OTHER IMPLICATIONS OF THE PRESENT HYPOTHESIS}

If the present hypothesis is correct, it has other significant implications in medicine and biology. Again, due to limited spaces, only a few important implications will be briefly discussed below.

The mechanism by which alcohols exert their intoxicating effect is not well understood $(58,59)$. However, it is known that ethanol and other short-chain alcohols are lipid-soluble and affect a variety of physiochemical structural properties of the biological membranes (see, e.g., 59). According to the present hypothesis, the intoxicating effect of alcohols is likely due to the cellular responses of the brain to mild hypoxia or hypoxia-mimic situations at subcellular or molecular level caused by the perturbation of oxygen pathways in membranes and proteins by these alcohols.

Second, the present hypothesis suggests the existence of endogenous modulating messengers responsible for producing general anesthesia through the oxygen-sensing mechanisms. Thus, some substances such as ketamine may produce anesthetic effect as messenger-mimic substances by direct binding to ion channels and receptors involved in brain functions. Ketamine is known to be a non-competitive antagonist of the NMDA receptor (60). If these endogenous messengers do exist, they may play very important roles in the normal functions of the brain such as regulations of sleep and consciousness. It has been speculated that the endogenous analogue of general anesthesia may exist (61).

Third, some general anesthetics have been shown to have neural protective effect against ischemia, but the underlying mechanisms are unknown $(62,63)$. It has been speculated that they produce such effect by acting as free radical scavengers (63). According to the present hypothesis, this effect is probably due to the perturbation of oxygen pathways in both membranes and proteins such that the neurons are in a depressed metabolic states and smaller amount of oxidative substances such as free radicals is generated upon reperfusion with oxygen.

Finally, many side effects of general anesthetics including some toxic effects may be explained according to the proposed mechanism. The key pathological condition to be considered under the present hypothesis is hypoxia or hypoxia-mimic situations at sub-cellular or molecular level. Thus, overdose or other misuse of general anesthetics may cause necrosis in brain, liver or other vital organs of the body. Indeed, some published results indicate that certain anesthetics cause necrosis in the liver (64). 


\section{CONCLUSION}

We have proposed in this paper a novel unitary mechanism of general anesthesia verifiable by experiments. In the proposed mechanism, general anesthetics perturb oxygen pathways in both membranes and oxygen-utilizing proteins such that its availabilities to the sites of utilization are reduced which in turn triggers cascading cellular responses through oxygen-sensing mechanisms resulting in general anesthesia. The present hypothesis provides a unified framework for explaining phenomena associated with general anesthesia and experimental results on the actions of general anesthetics. If valid, the proposed mechanism also has other significant medical and biological implications.

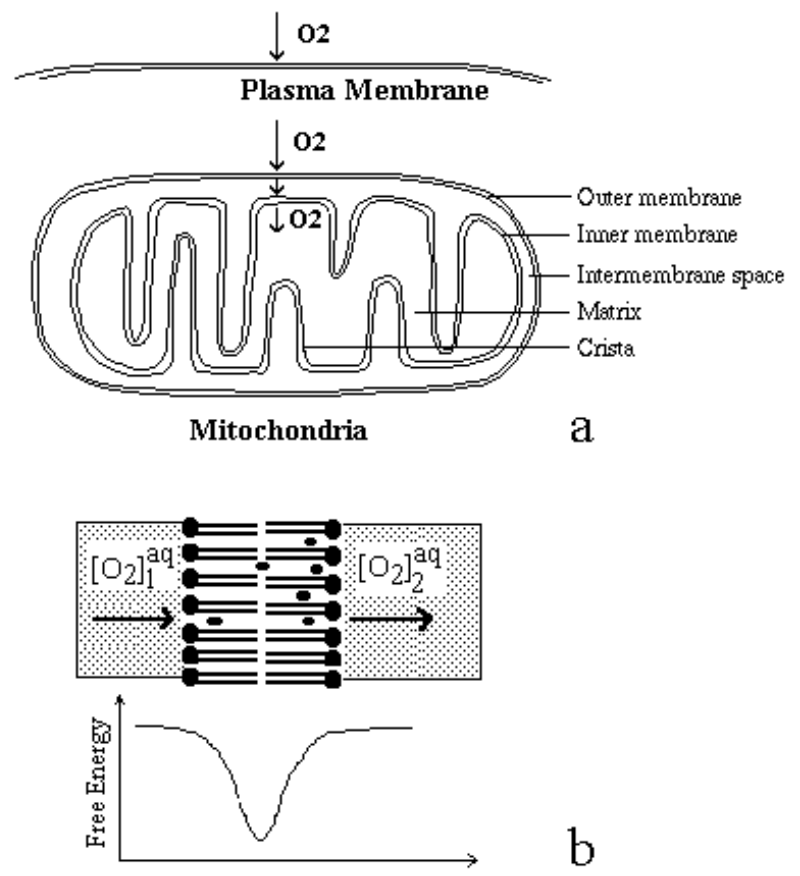

FIG. 1. Oxygen transport across cell membranes. Oxygen has to diffuse through the plasma membrane and the outer and inner membranes of the mitochondria before it can reach the mitochondrial matrix (a). At each membrane, oxygen has to diffuse through a first polar surface region, a hydrophobic region and a second polar surface region (b). The free energy barrier for oxygen uptake is schematically shown (b). 


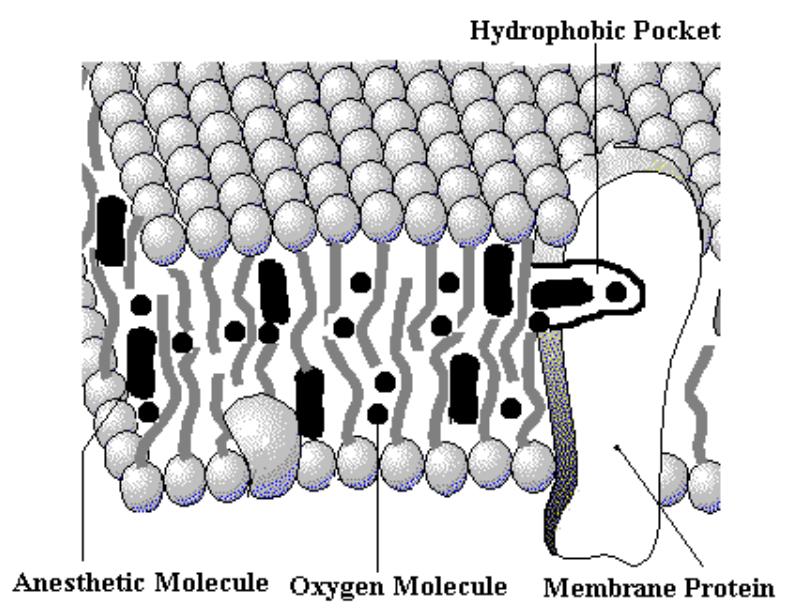

FIG. 2. Perturbation of oxygen pathways in membranes and proteins by general anesthetics. According to the present hypothesis, anesthetic molecules narrow, block, dislocate, distort or otherwise interfere with oxygen pathways in both membranes and proteins. They perturb not only oxygen transport across cell membranes but also its lateral movement within the membrane and the movement within a hydrophobic pocket of the protein.

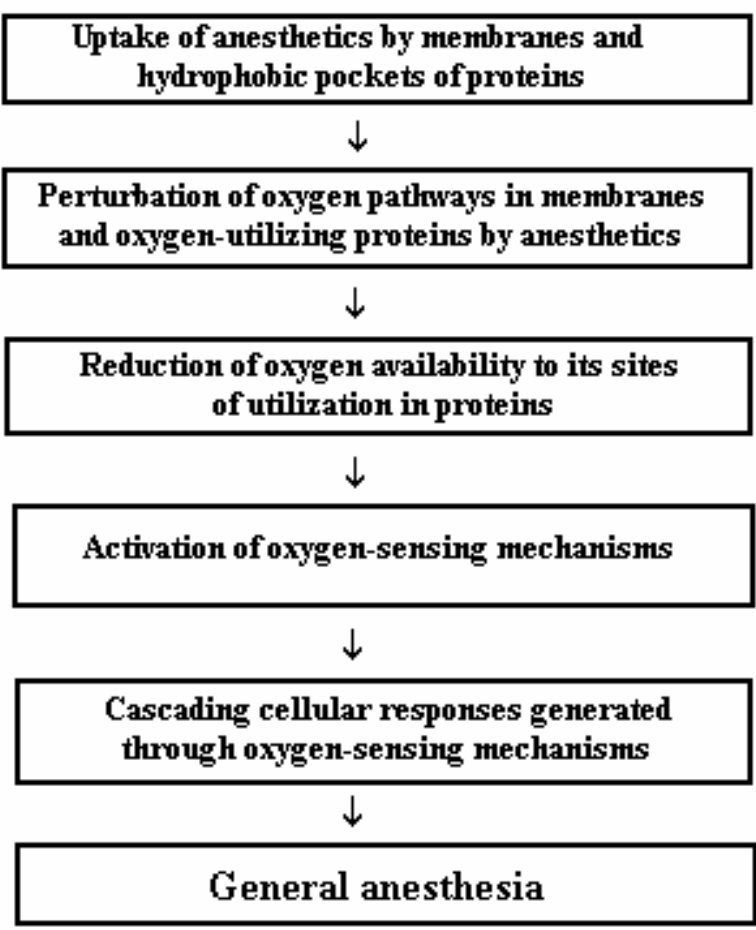


FIG. 3. Schematic diagram of the novel unitary mechanism of general anesthesia according to the present hypothesis.

\section{REFERENCES}

1. Scholz, A., Appel, N., Vogel, W. Two types of TTX-resistant and one TTX-sensitive Na channel in rat dorsal root ganglion neurons and their blockade by halothane. Eur. J. Neurosci. Suppl. 1998; 10: 2547-2556.

2. Zhang, L., Oz, M., Stewart, R. R., Peoples, R. W., Weight, F. F. Volatile general anaesthetic actions on recombinant nACh alpha 7, 5-HT3 and chimeric nACh alpha 7-5HT3 receptors expressed in Xenopus oocytes. Br. J. Pharmacol. 1997; 120: 353-355.

3. Krnjevic, K. Cellular and synaptic actions of general anaesthetics. Gen. Pharmacol. 1992; 23: 965-975.

4. Pancrazio, J. J. Halothane and isoflurane preferentially depress a slowly inactivating component of $\mathrm{Ca} 2+$ channel current in guinea-pig myocytes. J. Physiol. (London) 1996; 494: 91-103.

5. Harrison, N. L., Kugler, J. L., Jones, M. V., Greenblatt, E. P., Pritchett, D. B. Positive modulation of human gamma-aminobutyric acid type $\mathrm{A}$ and glycine receptors by the inhalation anesthetic isoflurane. Mol. Pharmacol. 1993; 44: 628-632.

6. Violet, J. M., Downie, D. L., Nakisa, R. C., Lieb, W. R., Franks, N. P. Differential sensitivities of mammalian neuronal and muscle nicotinic acetylcholine receptors to general anesthetics. Anesthesiology 1997; 86: 866-874.

7. Jenkins, A., Franks, N. P., Lieb, W. R. Actions of general anaesthetics on 5-HT3 receptors in N1E-115 neuroblastoma cells. Br. J. Pharmacol. 1996; 117: 1507-1515.

8. Kaech, S., Brinkhaus, H., Matus, A. Volatile anesthetics block actin-based motility in dendritic spines. Proc. Natl. Acad. Sci. U.S.A. 1999; 96: 10433-10437.

9. Meyer, H. H. Arch. Exp. Pathol. Pharmakol 1899; 42: 109-118.

10. Seeman, P. The membrane actions of anesthetics and tranquilizers. Pharmacol. Rev. 1972; 24: 583-655.

11. Trudell, J. R. A unitary theory of anesthesia based on lateral phase separations in nerve membranes. Anesthesiology 1977; 46: 5-10.

12. O'Leary, T. J. Effects of small nonpolar molecules on membrane compressibility and permeability. A theoretical study of the effects of anesthetic gases. Biophys. Chem. 1982; 15: $299-310$.

13. Miller, K. W., Firestone, L. L., Alifimoff, J. K., Streicher, P. Nonanesthetic alcohols dissolve in synaptic membranes without perturbing their lipids. Proc. Natl. Acad. Sci. U.S.A. 1989; 86: 1084-1087.

14. Cantor, R. S. The lateral pressure profile in membranes: a physical mechanism of general anesthesia. Biochemistry 1997; 36: 2339-2344.

15. Franks, N. P., Lieb, W. R. Molecular and cellular mechanisms of general anaesthesia. Nature (London) 1994; 367: 607-614.

16. Langmoen, I. A., Larsen, M., Berg-Johnsen, J. Volatile anaesthetics: cellular mechanisms of action. Eur. J. Anaesthesiol. 1995; 12: 51-58.

17. Harrison, N. L. Optical isomers open a new window on anesthetic mechanism. Anesthesiology 1998; 88: 566-568.

18. Magistretti, P. J., Pellerin, L, Martin, J.L. Brain Energy Metabolism: An Integrated Cellular Perspective in: Psychopharmocology, the Fourth Generation of Progress (Online Edition): http://www.acnp.org/g4/gn401000064/default.htm.

19. Weisiger, R.A. Impact of Extracellular and Intracellular Diffusion on Hepatic Uptake Kinetics in: Whole Organ Approach to Cellular Metabolism, Bassingthwaighte, J.B., Goresky, C. A., \& Linehan, J. H. Eds. (Springer Verlag, New York 1998) pp 389-423. 
20. Simon, S. A., Gutknecht, J. Solubility of carbon dioxide in lipid bilayer membranes and organic solvents. Biochim. Biophys. Acta 1980; 596: 352-358.

21. Kosztolowicz, T., Mrowczynski S. Membrane Boundary Condition LANL e-Print Archives 2000: http://xxx.lanl.gov/abs/cond-mat/0003177.

22. Subczynski, W.K., Hyde, J.S., Kusumi, A. Oxygen permeability of phosphatidylcholine-cholesterol membranes. Proc. Natl Acad. Sci. U. S. A. 1989; 86: 4474-4478.

23. Subczynski, W.K., Hopwood, L.E., Hyde, J.S. Is the mammalian cell plasma membrane a barrier to oxygen transport? J. Gen. Physiol. 1992;100: 69-87.

24. Kikeri, D., Sun, A., Zeidel, M. L., Herbert, S. C. Cell membranes impermeable to NH3. Nature 1989; 339:478-480.

25. Morse, P. D. $2^{\text {nd }}$, Swartz, H. M. Measurement of intracellular oxygen concentration using the spin label TEMPOL. Magn. Reson. Med. 1985; 2: 114-127.

26. Hu, H. P., Sosnovsky, G., Swartz, H. M. Simultaneous measurements of the intra- and extra-cellular oxygen concentration in viable cells. Biochim. Biophys. Acta 1992; 1112:161-166.

27. James, P. E., Grinberg, O. Y., Michaels, G., Swartz, H. M. Intraphagosomal oxygen in stimulated macrophages. J. Cell. Physiol. 1995; 163: 241-247.

28. Swartz, H. M. Measurements of intracellular concentrations of oxygen: experimental results and conceptual implications of an observed gradient between intracellular and extracellular concentrations of oxygen. Adv. Exp. Med. Biol. 1994; 345: 799-806.

29. Kaibara, M., Tsong, T. Y. Voltage pulsation of sickle erythrocytes enhances membrane permeability to oxygen. Biochim. Biophys. Acta 1980; 595:146-150.

30. Jones, D. P., Kennedy, F. G. Intracellular oxygen supply during hypoxia. Am J Physiol 1982; 243: C247-253.

31. Richards, C. D., Martin, K., Gregory, S., Keightley, C. A., Hesketh, T. R., Smith G. A., Warren G. B., Metcalfe, J. C. Degenerate perturbations of protein structure as the mechanism of anaesthetic action. Nature 1978; 276:775-779.

32. Lee, A. G. Interactions between anesthetics and lipid mixtures. Normal alcohols. Biochemistry 1976; 15: 2448-1454.

33. Chiou, J. S., Ma, S. M., Kamaya, H., Ueda, I. Anesthesia cutoff phenomenon: interfacial hydrogen bonding. Science 1990; 248: 583-585.

34. Franks, N. P., Lieb, W. R. Do general anaesthetics act by competitive binding to specific receptors? Nature 1984; 310:599-601.

35. Hasinoff, B. B., Davey, J. P. The inhibition of a membrane-bound enzyme as a model for anaesthetic action and drug toxicity. Biochem. J. 1989; 258: 101-107.

36. Eckenhoff, R. G., Johansson, J. S., Molecular interactions between inhaled anesthetics and proteins. Pharmacol. Rev. 1997; 49: 343-367.

37. Chandel, N.S., Schumacker, P. T. Cellular oxygen sensing by mitochondria: old questions, new insight. J. Appl. Physiol. 2000; 88:1880-1889.

38. Lahiri, S. Historical perspectives of cellular oxygen sensing and responses to hypoxia. $J$. Appl. Physiol. 2000; 88: 1467-1473.

39. Prabhakar, N. R. Oxygen sensing by the carotid body chemoreceptors. J. Appl. Physiol. 2000; 88: 2287-2295.

40. Hochachka, P. W., Buck L. T., Doll, C. J., Land, S. C. Unifying theory of hypoxia tolerance: molecular/metabolic defense and rescue mechanisms for surviving oxygen lack. Proc. Natl. Acad. Sci. U. S .A. 1996; 93: 9493-9498.

41. Jiang C., Haddad, G. G. A direct mechanism for sensing low oxygen levels by central neurons. Proc. Natl. Acad. Sci. U. S. A. 1994; 91:7198-7201.

42. Whalen, W. J. Riley J., Nair, P. A microelectrode for measuring intracellular PO2. J. Appl Physiol 1967; 23:798-801.

43. Whalen, W. J. Intracellular oxygen microelectrodes. Adv. Exp. Med. Biol. 1973; 37A: 17-22.

44. Longmuir, I. S., Knopp, J. S. A new histochemical stain for intracellular oxygen. Adv. Exp. Med. Biol. 37A: 55-57. 
45. Benson, D. M., Knopp, J. A., Longmuir, I. S. Intracellular oxygen measurements of mouse liver cells using quantitative fluorescence video microscopy. Biochim. Biophys. Acta 1980; 591: 187-197.

46. Sugano, T., Oshino, N., Chance, B. Mitochondrial function under hypoxic conditions: the steady states of cytochrome alpha+alpha3 and their relation to mitochondrial energy states. Biochim. Biophys. Acta 1974; 368: 298-310.

47. Chance, B., Oshino, N., Sugano, T., Mayevsky, A. Basic principles of tissue oxygen determination from mitochondrial signals. Adv. Exp. Med. Biol. 1973; 37A: 277-292.

48. Chance, B., Barlow, C., Nakase, Y., Takeda H., Mayersky, A., Fischette, R., Graham, N., Sorge, J. Heterogeneity of oxygen delivery in normoxic and hypoxic states: a fluorometer study. Am J Physiol 1978; 235: H809-820.

49. Chen, K., Morse, P. D. $2^{\text {nd }}$, Swartz, H. M. Kinetics of enzyme-mediated reduction of lipid soluble nitroxide spin labels by living cells. Biochim. Biophys. Acta 1988; 943: 477-484.

50. Chen, K., Glockner, J. F., Morse, P. D. $2^{\text {nd }}$, Swartz, H. M. Effects of oxygen on the metabolism of nitroxide spin labels in cells. Biochemistry 1989; 28: 2496-2501.

51. Shulman, R. G., Rothman, D. L., Hyder, F. Stimulated changes in localized cerebral energy consumption under anesthesia. Proc. Natl. Acad. Sci. U.S.A.1999; 96: 3245-3250.

52. Hodge, R. D., Atkinson, J., Gill, B., Crelier, G. R., Marrett, S., Pike, G. B. Linear coupling between cerebral blood flow and oxygen consumption in activated human cortex. . Proc. Natl. Acad. Sci. U.S.A.1999; 96: 9403-9408.

53. Saiki, C., Mortola, J. P. Effect of 2,4-dinitrophenol on the hypometabolic response to hypoxia of conscious adult rats. J. Appl. Physiol. 1997; 83: 537-542.

54. Rohlicek, C. V., Saiki, C., Matsuoka, T., Mortola, J. P. Oxygen transport in conscious newborn dogs during hypoxic hypometabolism. J. Appl. Physiol. 1998; 84: 763-768.

55. Winter, P. M., Smith, R. A., Smith, M., Eger, E. I. $3^{\text {rd }}$ Pressure antagonism of barbiturate anesthesia. Anesthesiology 1976; 44: 416-419.

56. Mihic, S. J., Ye, Q., Wick, M. J., et al. Sites of alcohol and volatile anaesthetic action on GABA(A) and glycine receptors Nature 1997; 389: 385-389.

57. Mascia, M. P., Trudell, J. R., Harris, R. A. Specific binding sites for alcohols and anesthetics on ligand-gated ion channels. Proc.Natl.Acad.Sci.U.S.A. 2000; 97: 93059310.

58. Lovinger, D. M., White, G., Weight, F. F. Ethanol inhibits NMDA-activated ion current in hippocampal neurons. Science 1989; 243:1721-1724.

59. Kyeder, M., Pifat, G., Jeloyecki, A., Klai, B., Pecar, S., Schara, M. The EPR study of LDL perturbed by alcohols with different molecular architecture. Alcohol 2000; 21: 141147.

60. Adams, H. A., Werner, C. From the racemate to the eutomer: (S)-ketamine. Renaissance of a substance? Anaesthesist 1997; 46:1026-1042.

61. Lerner, R. A. A hypothesis about the endogenous analogue of general anesthesia. Proc. Natl. Acad. Sci. U. S. A. 1997; 94: 13375-13377.

62. Mantz, J. Neuroprotective effects of anesthetics. Ann. Fr. Anesth. Reanim. 1999; 18: 588592.

63. Almaas, R., Saugstad, O. D., Pleasure, D. \& Rootwelt, T. Effect of Barbiturates on Hydroxyl Radicals, Lipid Peroxidation, and Hypoxic Cell Death in Human NT2-N Neurons. Anesthesiology 2000; 92:764-774.

64. Berthoud, M. C., Reilly, C. S., Adverse effects of general anaesthetics. Drug Saf. 1992; 7: 434-459. 